\title{
Channels in a porous scaffold: a new player for vascularization
}

\author{
Yunqing Kang ${ }^{* 1,2} \&$ Jia Chang ${ }^{3}$ \\ ${ }^{1}$ Department of Ocean \& Mechanical Engineering, College of Engineering and Computer Science, Florida Atlantic University, Boca \\ Raton, FL 33431, USA \\ ${ }^{2}$ Department of Biomedical Science, College of Medicine, Florida Atlantic University, Boca Raton, FL 33431, USA \\ ${ }^{3}$ Department of Periodontology, University of Florida College of Dentistry, Gainesville, FL 32610, USA \\ *Author for correspondence: Tel.: +1 561297 3943; kangy@fau.edu
}

\begin{abstract}
Vascularization is essential for tissue regeneration. Despite extensive efforts in the past decades, sufficient and rapid vascularization remains a major challenge in tissue engineering. Many studies have shown that the addition of channels in a porous scaffold can provide the ability to promote cell growth and rapid vascularization, thus leading to better outcomes in new tissue formation. Large size scaffolds lack perfusable channel networks and negatively impair the survival of transplanted cells and tissue function development, leading to necrotic core formation and the failure of functional tissue formation. Presently, there are many methods to produce channels in porous scaffolds for vascularization. Here, we review the function of channels in porous scaffolds and the approaches to produce those channels.
\end{abstract}

First draft submitted: 2 March 2018; Accepted for publication: 6 August 2018; Published online: 24 September 2018

Keywords: 3D printing $\bullet$ microchannels $\bullet$ regenerative medicine $\bullet$ scaffold $\bullet$ tissue engineering $\bullet$ vascularization

\section{Vascularization in tissue engineering}

Synthetic tissue-engineered scaffolds hold great promise for tissue regenerative therapy without the undesirable side effects of autografts or allografts, such as limited supply, donor site morbidity, prolonged recovery and pathogen transfer of the grafts [1-3]. Tissue-engineered scaffolds have been extensively studied in the past decades [4-6]. However, insufficient vascularization in the entire implanted scaffolds hinders the successful transplantation of porous scaffolds for bone, cartilage and cardiac tissue repair [7-11].

Several strategies have been developed for enhancing rapid vascularization and instant blood perfusion in implanted scaffolds [12-14]. These methods include using angiogenic growth factors [7,15] and coculturing pericytes and endothelial cells on scaffolds [16-18]. Although these strategies can initialize angiogenesis and growth of blood vessels, they can only promote blood vessels to grow a short distance from the surface of the implants. New blood vessels grow too slowly to provide instant blood perfusion to the center of the implanted constructs [7]. One major hindrance may be the spatial challenges posed by porous scaffolds, which prevent host vasculature and nutrient transportation from invading and diffusing rapidly into the center zone [19]. Although interconnected pores are essential, and are also required to facilitate nutrient diffusion and cell migration, pores alone cannot provide enough space and a clear path, and are insufficient for the in-growth of host vasculature and nutrient diffusion [19,20]. The vascular system provides most tissues in our body with a supply of nutrients and oxygen and ensures proper waste removal. Considering that the maximum distance between capillaries is around $200 \mu \mathrm{m}$ [21], oxygen and nutrients cannot be sufficiently supplied to a large-sized construct through pores alone, which leads to necrosis and tissue death in the center of the implanted construct [22-24]. When cells grow beyond the diffusion range of oxygen, hypoxia initiates the invading of new blood vessels. However, blood vessels require several weeks to invade a millimeter-sized implant for complete vascularization. In this case, pores and biological growth factors are not sufficient. Scaffold structures like channels imitating blood vessels or facilitating the invasion of blood vessel become critical $[7,25]$. In this paper, we review the importance of channels in a porous scaffold for tissue regeneration. 


\section{The theoretical support of using channels for vascularization}

Tissue engineering strategy utilizes a porous scaffold, growth factor and cells for tissue regeneration [26]. The prerequisite condition for success is the harmonious cooperation of the three components. However, despite the recent significant progress in tissue engineering, many challenges stand between the current porous scaffold and its therapeutic implementation in humans. One of the most critical issues in cell survival and growth is insufficient mass transport, which leads to slow diffusion of oxygen and nutrients in the center of a thick porous scaffold [27,28]. The low diffusion ability of a thick porous scaffold makes cells in the central region suffer from insufficient nutrient and oxygen supply, and waste products accumulate within the scaffolds, which cause cell death or heterogeneous cell distributions in the scaffold [29,30].

Channels have the potential to regulate the distribution of chemicals in porous scaffolds. Mashayekhan and colleagues established a mathematic model to elucidate the theoretical basis of the role of channels in cell growth [31]. The simulation results based on the mathematical model showed that oxygen concentration decreased significantly along with the length of the construct, but with the increase of the channel numbers, the average cell density increased by $30 \%$ of the initial seeded cell density. Their model demonstrated that the increase of the channel number within the scaffold significantly increased the cell density. Another simulation model has been established to investigate the effect of a parallel channel array in a porous poly(glycerol sebacate) scaffold on the oxygen distribution in the construct [32]. The parallel channel array mimics the in vivo capillary tissue bed. Results showed that the channel was favorable for high cell density. In the channel, the primary modes of mass transfer were axial convection and radial diffusion. In the tissue region, the mass transfer was axial and radial diffusion [32]. In Khademhosseini and colleagues' study, they engineered a 3D model for cell-laden microfluidic channels [33]. Their model system simulated theoretical nutrient diffusion in a cell-laden hydrogel. They compared the spatial distribution of viable cells within the 3D hydrogel without channels and with single- and dual-perfusion microfluidic channels. Results found that, no matter how many channels were in the hydrogel construct, channels increased nutrient and oxygen transport as a way to improve cell viability, compared with the hydrogel construct without channels. This theoretical model demonstrated that there was a close correlation between diffusive nutrient distribution and cell viability throughout the hydrogel construct. The simulation results showed that it is necessary to have channels spaced closely to avoid oxygen transport limitations, as the oxygen concentration was higher in the lumen of the parallel channels than that in the surrounding space [34]. These mathematic models successfully simulated the effect of channels on oxygen distribution in the porous scaffolds, which resulted in a higher cell density in the channel space. Therefore, channels played a promoting role in cell growth and vascularization.

\section{Channel types in different porous scaffolds \\ Single/multiple channels in hydrogels}

To provide sufficient oxygen and nutrients to a tissue-engineered construct, many studies made efforts to mimic the lumen structure of blood vessels by creating microvessel-like channels in the constructs. Takei $e t$ al. used alginate hydrogel microfibers to assemble vascular-like microchannels [35]. Their study found that the cell culture medium could flow through the channels quickly (within $10 \mathrm{~min}$ ) after the immobilization of the cells in the assembly gel. The human hepatoblastoma cells proliferated in the gel portions of the microfibers and maintained their specific function during the perfusion culture of 7 days. The novel mimicked vascular-like networks seemed to have the potential to allow the creation of engineered tissues in vitro. When implanted in vivo, the channeled hydrogel promoted cell infiltration. For the survival of engineered tissues in vivo, vascularization is critical, and channels, as a physical structure, provide the entrance for the in-growth of blood vessels. This conclusion was further confirmed in Mao and colleagues' study [36]. They created three microchannels with $1 \mathrm{~mm}$ of diameter in poly(ethylene glycol) diacrylate (PEG) hydrogel cylinders. The results from the in vivo subcutaneous implantation experiment indicated that there was no obvious infiltrating host tissue observed if PEG hydrogel did not contain microchannels. On the contrary, host tissue infiltration was apparently observed when PEG hydrogel cylinders contained three microchannels. Interestingly, the host tissue infiltration was found primarily in the lumen of the microchannels, but scarcely in the rest of PEG hydrogel. The infiltrating host tissue included vascular structures with erythrocyte-filled blood vessels that were lined by endothelial cells [36]. This study directly showed the importance of microchannels in a hydrogel construct for in vivo vascularization. In another study, microchannels in a hydrogel promoted nerve repair. The results of the study showed that the multiple channels in the scaffold-guided axonal regeneration [37]. The tissue regenerative ability may result from the functionality of vascular-like microchannels, which facilitated the mass transport. Khademhosseini and colleagues' study proved that this kind of functionality 

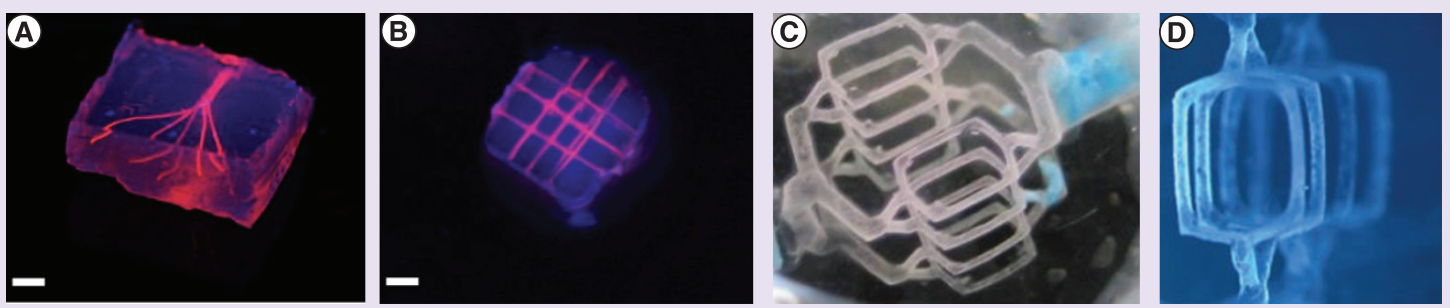

Figure 1. Microchannels in a hydrogel construct prepared by using the combination method of a sacrificial agarose material template and 3D bioprinting. Branch channels (A) and lattice channels (B) in a gelatin-modified hydrogel (scale bars $=3 \mathrm{~mm}$ ). A gelatin template without acetone wash, displaying three dimensionality of the network (C \& D). (A \& B) Re-edited and reproduced with permission from [38].

(C \& D) Re-edited and reproduced with permission from [44].

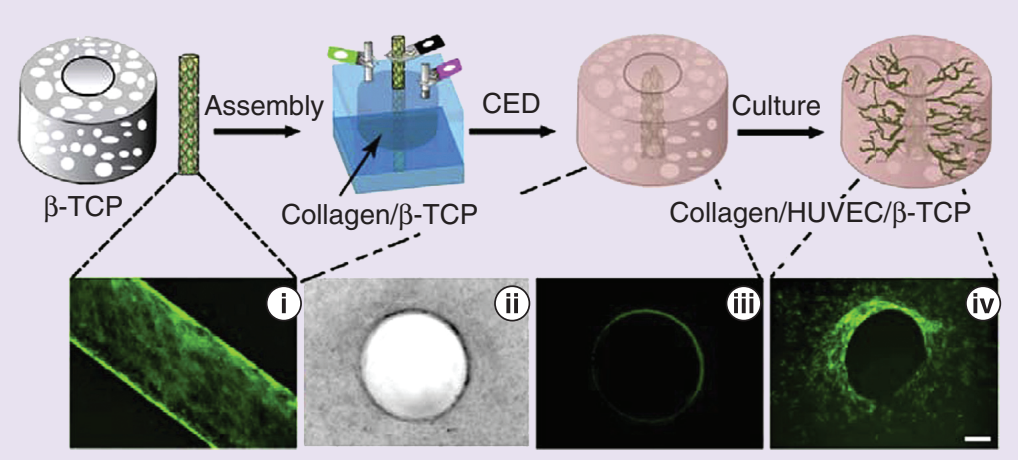

Figure 2. Channels in a $\beta$-tricalcium phosphate scaffold made by the mechanical removal spacer method. Schematic diagrams show the assembly procedures of a microchannel in a type I collagen hydrogel and in a $\beta$-TCP graft by using a cell electrochemical detachment technique. (A) A fluorescent image of HUVECs on an oligopeptide-coated rod, (B) a bright-field image of the microchannel in the collagen hydrogel (top view), (C) a fluorescent image of the microchannel in the collagen and (D) a fluorescent image of networks sprouting from the microchannel into the surrounding area of the graft (scale bar $=100 \mu \mathrm{m}$ ).

$\beta$-TCP: $\beta$-Tricalcium phosphate scaffold; CED: Cell electrochemical detachment; HUVEC: Human umbilical vein endothelial cell.

Re-edited and reproduced with permission from [39].

of fabricated vascular networks embedded in hydrogels. In Khademhosseini and colleagues' study, the vascular-like microchannels were fabricated by 3D-bioprinted sacrificial materials and embedded in a hydrogel (Figure 1A-D). This kind of microchannel promoted mass transport, cellular viability and differentiation [38].

Microchannels in a hydrogel construct provide not only a tubular structure to facilitate nutrition transport but also a luminal tube for endothelial cells to align on the wall surface to form an endothelium (Figure 2) [39-41]. Thus, a microchannel becomes a functional vascular network, not just a physical support. The cells attached to the wall of the microchannels in a hydrogel construct grew and invaded into the hydrogel to form new networks for vascularization.

Besides parallel microchannels array or single/dual straight channels in hydrogels, branched microchannels and complex microchannels networks had been developed to mimic vascular system (Figure 1C \& D) [42-44]. A complex $3 \mathrm{D}$ vascular-like multiscale system that was composed of larger vascular perfusion channels promoted the growth of a capillary network and provided a feasible and promising approach to address the vascularization issue [45]. The multiscale vascular system fabricated by using a $3 \mathrm{D}$ bioprinting technique significantly promoted the formation of a capillary network [45]. Additionally, the bioprinting technology allowed cells to be easily encapsulated into the $3 \mathrm{D}$ matrix within a scaffold at the desired location [46]. 
Single/multiple channels in polymer/ceramic porous scaffolds

Many studies focused on embedding microchannels in cross-linked hydrogels, as hydrogels are biocompatible and shapeable to encapsulate cells. Based on these advantages of a hydrogel, the addition of channels further enhances the function of the hydrogel in vascularization. For polymers or bioceramic porous scaffolds, hollow channels also brought a promising platform for integrating vascularization strategies in a single construct. For example, in a calcium phosphate cement material, interconnected hollow channels exhibited favorable characteristics for vascularization [47]. The degree of vascularization in vivo was different based on different channel sizes. Interconnected multiscale channels promoted the ingrowth of blood vessels from host to scaffold through channels [47]. Apparently, the scaffold with micropatterned channels favored the penetration of blood vessels throughout the newly formed tissue, as well as in the surrounding host tissue [48]. Kaplan and colleagues' studies found that multiple channels in porous silk scaffolds promoted the survival of transplanted cells in vivo [49]. For acellular scaffolds, hollow channels with $254 \mu \mathrm{m}$ diameter not only improved endothelial cell survival and in vitro prevascularization but also promoted in vivo initial integration and tissue ingrowth. Coculturing endothelial cells in the hollow channels additively promoted rapid vascularization and perfusion in vivo and synergistically promoted tissue ingrowth [49]. These results proved that silk scaffolds with multiple channels of $254 \mu \mathrm{m}$ had the functionality to promote tissue regeneration. These channels also provided conduits for medium perfusion and efficient transport of oxygen to the cells, by a combination of perfusable flow and molecular diffusion over short distances between the channels. In another study [50], a porous elastomeric scaffold with an array of channels promoted high efficiency $(77.2 \pm 23.7 \%)$ of cell seeding when seeded with neonatal rat cardiac myocytes. Uniform spatial cell distributions were obtained when the scaffold had channels for perfusion seeding.

Channels with cells can be utilized to engineer thick tissue-engineered constructs. As indicated in our previous published studies [39], we used our template-casting method to create a single macrochannel $(3 \mathrm{~mm})$ in a porous $\beta$-tricalcium phosphate ( $\beta$-TCP) scaffold, and integrated a cell-lined collagen channel inside (Figure 2$)$. The channeled $\beta$-TCP scaffold integrated with cell-lined collagen microchannel was implanted in the subcutaneous pocket of a mouse. Interestingly, we found that host vasculature first grew into the channel to form blood flow and then into the other surrounding porous areas of the scaffold ( $8 \mathrm{~mm}$ diameter). Interestingly, it took much longer for a porous scaffold without a channel to form blood flow [39]. These results suggest that a channel efficiently improved the nutrition diffusion and blood flow to the central zone of the porous scaffold and facilitated sufficient vascularization [39,51]. Based on these results, it is plausible to conceive that an array of hollow macrochannels in a porous scaffold could robustly facilitate nutrient transportation and instant blood perfusion. In our new study [52], we implanted porous $\beta$-TCP scaffolds with multiple channels $(1 \mathrm{~mm}$ diameter each channel) into mandibular bone defects of beagle dogs. Results showed that the vertical hollow channels accelerated the degradation of the $\beta$-TCP scaffolds. More volume of newly formed bone tissues with more blood vessels were augmented in the channeled scaffolds. Channeled scaffolds significantly promoted the new bone formation and increased the height of the mandible. These findings indicated that channeled scaffolds facilitated vascularization and bone formation [52].

Although the size and number of the channels in different scaffolds were different, all these studies showed that channel played a critical role in vascularization. Channel types related details are listed in Table 1.

\section{Approaches of channel fabrication}

To create perfusable channels in hydrogels, polymers or ceramic scaffolds, many strategies have been developed.

\section{Mechanically removable templates}

Using templates or removable rods can generate defined channels. The channel diameter and space in hydrogels, polymers and ceramic porous scaffolds can be controlled by the dimensions of the templates [39,49,53]. Figure 3 shows the schematic procedures for the preparation of channels in a scaffold using a template of spacers. Kaplan and colleagues used linear wire arrays to make porous silk scaffolds with 254 or $508 \mu \mathrm{m}$ diameter of vertical channels [53]. Kang and colleagues used templating rods to make $\beta$-TCP scaffolds with five channels of $1 \mathrm{~mm}$ diameter [52]. The template spacer strategy showed the potential to not only create channels in the porous scaffold but also provide the structure for cells to align in the formatted channels [54]. To vascularize a tissue-engineered construct, endothelial cells are commonly used. To align endothelial cells on the wall of the channels, cell suspension was injected into the formed channels, and endothelial cells then attach on the inner wall surface of channels. The attached cells in the channels can grow and sprout out into the surrounding area of hydrogels for the vascularization of the entire construct. Fukuda and colleagues developed a unique technique to realize the creation of channels and 
Table 1. Channel types in different scaffold types and their functions.

\begin{tabular}{|c|c|c|c|}
\hline Channel type (number, size) & Scaffold type (porous size) & Function results & Refs. \\
\hline $200,50 \mu \mathrm{m}$ & Alginate hollow microfibers & Microfibers maintained their specific function of HepG2 & [35] \\
\hline $3,1 \mathrm{~mm}$ & PEG hydrogel & $\begin{array}{l}\text { Host cell infiltrated into the lumen of the channel not in the other } \\
\text { part }\end{array}$ & [36] \\
\hline $7,250 \mu \mathrm{m}$ or $14,500 \mu \mathrm{m}$ & Agarose/collagen fibers & Microchannels promoted fascicular-like nerve repair & [37] \\
\hline $6,500 \mu \mathrm{m}$ & Methacrylated gelatin hydrogel & Form an endothelium aligned in the wall surface of the channel & [38] \\
\hline $1,600 \mu \mathrm{m}$ & Collagen hydrogel & Form functional vascular networks & [39-41] \\
\hline Branches, $500 \mu \mathrm{m}$ & Alginate hydrogel & Perfusable channel for vascularization & [42-44] \\
\hline $2,1 \mathrm{~mm}$ & Fibrin hydrogel & Multiscale vascular network and capillary formed within a fibrin & [45] \\
\hline 130 tetrahedrons, $1-2 \mathrm{~mm}$ & Polycaprolactone $(100-200 \mu \mathrm{m})$ & Support liver cells' growth and function & [46] \\
\hline $2,(255$ and $507 \mu \mathrm{m})$ & Calcium phosphate cement & $\begin{array}{l}\text { Pre-established vascular networks in CPC material anastomose with } \\
\text { host vessels }\end{array}$ & [47] \\
\hline $500 \mu \mathrm{m}$ & Chitosan scaffold $(600-900 \mu \mathrm{m})$ & Micropatterned scaffolds enhanced neovascularization & [48] \\
\hline Array, $250 \mu \mathrm{m}$ & $\begin{array}{l}\text { Poly(glycerol sebacate) scaffold } \\
(75-150 \mu \mathrm{m})\end{array}$ & Preferential cell attachment at the channel surfaces & [50] \\
\hline $5,1 \mathrm{~mm}$ & $\begin{array}{l}\text { Tricalcium phosphate scaffold } \\
(500 \mu \mathrm{m})\end{array}$ & Promoted blood vessels and bone formation & {$[51,52]$} \\
\hline
\end{tabular}

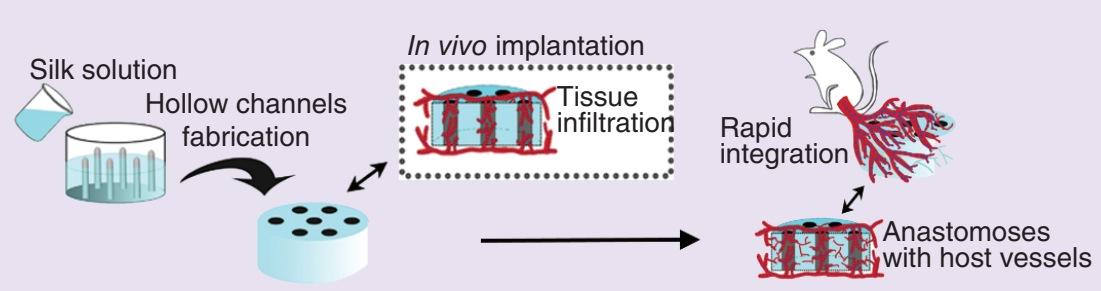

Figure 3. A schematic graph shows the experimental protocol for the vascularization of hollow channeled porous silk scaffolds. Host tissue rapidly infiltrated into the prefabricated arrayed channels. The in vitro incubated vascular networks rapidly anastomosed with the infiltrated host blood vessels.

Re-edited and reproduced with permission from [49].

cell alignment of the channels in a hydrogel construct in one step (Figure 4) [55]. Their approach involves using a glass rod to create a microchannel in a hydrogel $[40,56]$. A glass rod with a defined diameter was coated by a gold layer, then immersed in a peptide solution. The sequence of the peptide was particularly designed. Endothelial cells were seeded onto the surface of the peptide-coated rod. The cell-rod was then inserted into a mold and a collagen solution was casted into the mold. After the collagen solution was solidified, an electric current was applied onto the rod. The electric current cleaved the peptide, resulting in cell detachment. The detached cell layer was transferred to the surrounding wall of the channel in the hydrogel at the same time. The rod was then removed, leaving endothelial cell-lined vascular-like channels structure that was precisely aligned in the channel wall within the hydrogel $[40,57]$.

\section{'Bottom-up' modular technique}

Besides microchannels alone in a hydrogel construct, cells can be encapsulated into the hydrogel to vascularize the entire construct. In this case, the channels in the hydrogel facilitate the nutrient transport to the encapsulated cells and the infiltration of host cells. In Khademhosseini and colleagues' study [58], a 'bottom-up' tissue engineering approach was used to build cell-laden microengineered hydrogel (microgel) constructs embedded with vascular-like microchannels. Endothelial cells and smooth muscle cells were encapsulated into the microgel units with spatial arrangement mimicking the vascular physiology, which were then assembled into a blood vessel-like microchannel structure. Such a 'bottom-up' micromodular technique was often used to fabricate assembly microchannels within cell-laden hydrogels [38]. 


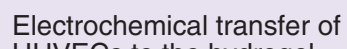
HUVECs to the hydrogel

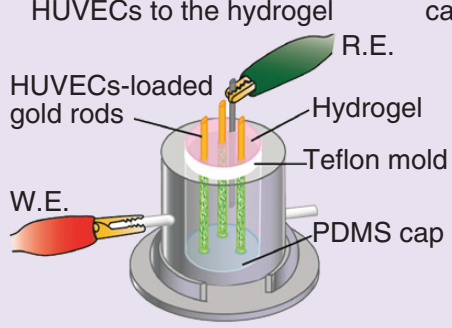

Addition of a PDMS cap and removal rods
3

Hydrogel removal from

the aluminium chamber
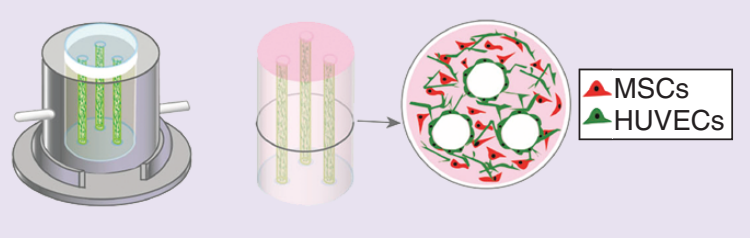

Figure 4. A schematic graph shows the fabrication procedure of a thick microchanneled fibrin hydrogel in a custom-made aluminum chamber. Gold-coated rods $(\varnothing 600 \mu \mathrm{m})$ attached with HUVECs were placed in a Teflon mold $(8 \mathrm{~mm} \mathrm{~A}-6.35 \mathrm{~mm})$ and hydrogel was casted. After gelation, HUVECs were electrically $(-1.0 \mathrm{~V})$ transferred to the microchannels within the hydrogel. A three-hole PDMS cap was placed on top of the construct and rods were removed.

HUVEC: Human umbilical vein endothelial cell; MSC: Mesenchymal stem cell; PDMS: Polydimethylsiloxane. Re-edited and reproduced with permission from [41].

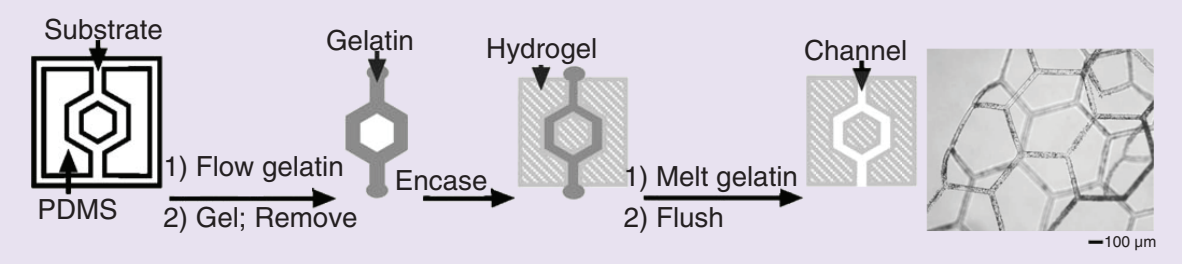

Figure 5. A schematic diagram shows the fabrication of microfluidic gels. Sealing a PDMS stamp to a substrate (glass or preoxidized PDMS) created a microfluidic network. Sequential introduction of a pluronic (1-6\% in PBS) and a liquid gelatin into the channels, and gelation at $4{ }^{\circ} \mathrm{C}$ for $15 \mathrm{~min}$ and at $23^{\circ} \mathrm{C}$ for $2 \mathrm{~h}$, yielded a gelatin mesh that was easily separated from the channels. Encapsulation of the mesh in a liquid hydrogel precursor (type I collagen, fibrinogen, Matrigel), followed by polymerization of the precursor and flushing at $37^{\circ} \mathrm{C}$, yielded a hydrogel with open microchannels.

PBS: Phosphate-buffered saline; PDMS: Polydimethylsiloxane.

Re-edited and reproduced with permission from [63].

\section{Sacrificial template}

Although removable template spacers can be involved in producin g channels in a porous scaffold or a hydrogel construct, this method can create only unbranched channels, which do not resemble the in vivo vasculatures. To mimic vascular-like branched channels, a sacrifice template method has been developed and widely used in the field [59]. This technique utilizes a sacrifice vascular-like model to form perfusable-branched microchannels. The structure of the template mimics the complex network of blood vessels. After the template was made, the precursor solution of a hydrogel was cast and then cross-linked. The template was then melted or dissolved, leaving branched microchannels inside the hydrogel $[38,60]$. For example, alginate serves as a sacrificial material and then collagen hydrogel was cast into the mold. Alginate material was then dissolved, leaving a complex channel network in the cross-linked collagen hydrogel [61]. Besides alginate materials [62], gelatin often serves as a sacrificial material for channel production, as gelatin material is a solid state at low temperature and becomes liquid at body temperature $[47,63]$. Utilizing this advantage of a gelatin material, gelatin can be made into all kinds of vascular-like branches for perfusable channels. For example, gelatin can be made into mesh network for channeled collagen constructs (Figure 5) [63]. Gelatin can be made into gelatin fibers and embedded in calcium phosphate cement and incubated [47]. Hollow-channeled calcium phosphate cement was then produced in vivo after the gelatin fibers dissolved. This channel fabrication methodology was reproducible and straightforward. These studies showed that sacrificial template method has been widely used to build perfusable vascular-like architectures in 


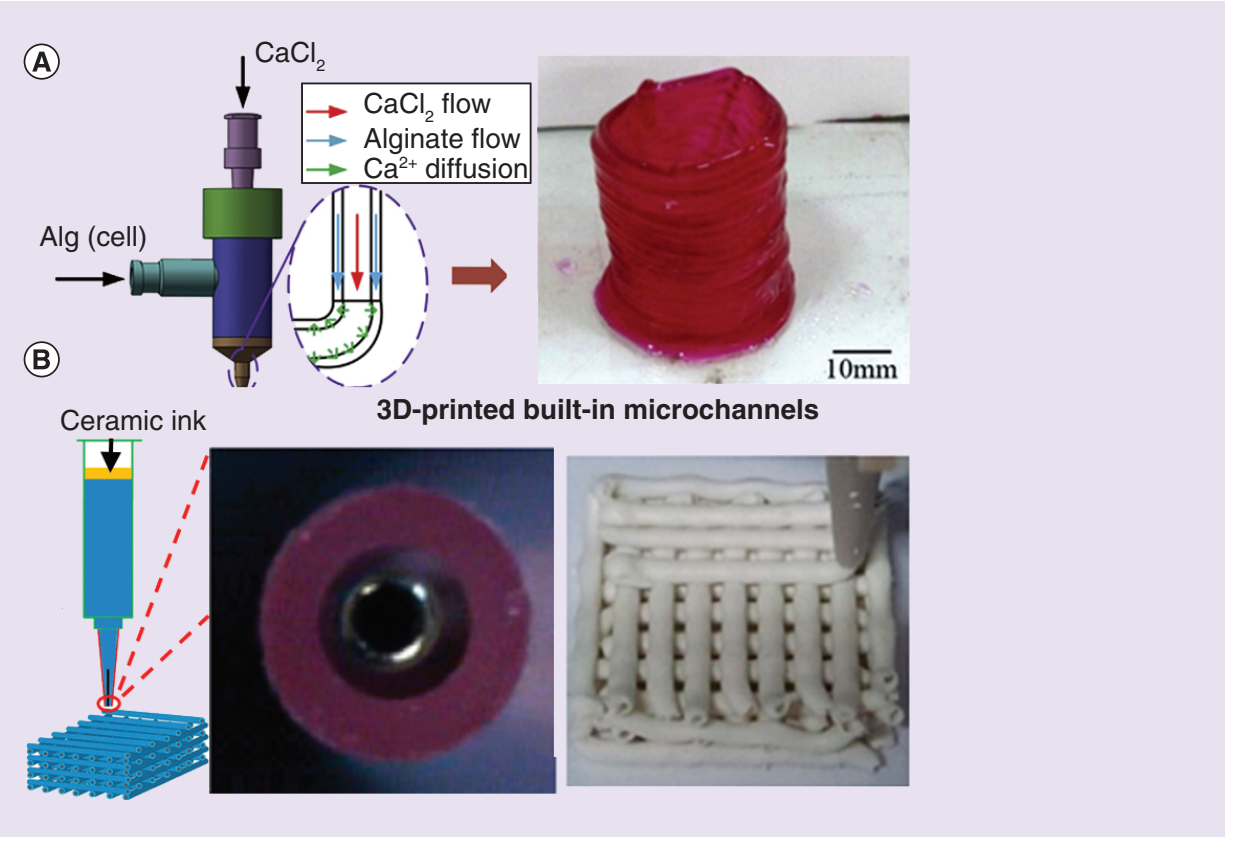

Figure 6. 3D-printed microchannels in hydrogel and ceramic scaffolds. A fabrication process of a 3D alginate structure with built-in microchannels (A); A procedure shows the fabrication of channeled bioceramic scaffolds: mixing bioceramic powders with F-127 solution (concentration: $20 \mathrm{wt} \%$ ) and alginate, printing the bioceramic inks via the coaxis nozzle (B).

(A) Re-edited with permission from [66].

(B) Re-edited and reproduced with permission from [69] (c) American Chemical Society (2015).

many different types of hydrogels [43,64]. However, most of these sacrificial templates had a 2D plane-connected network, not a 3D network (Figure 5). A new fused-deposit extrusion 3D filament printing system was developed to fabricate a hydrogel construct with a 3D perfusable-connected network. The perfusable channel network was molded into tree branches [42,65]. Once a complex-branched sacrificial template is printed, hydrogels were casted and then the sacrificial templates were melted or dissolved to form branched vascular-like channeled constructs for vascularization.

\section{D bioprinting}

Although sacrificial template can create microchannels in hydrogels, several steps are required to build up the templates and then make channels. New 3D bioprinting technology has been developed to directly produce channeled scaffolds for tissue engineering. For example, using coaxial nozzle-assisted 3D bioprinting to print hollow alginate fibers for built-in microchanneled scaffolds has been developed (Figure 6A) [66]. Using 3D printing technique to produce porous hydroxyapatite and TCP blocks with a central channel had also been successfully developed for vessel pedicle or nerve integration [67]. A rapid prototyping technology, fused deposition modeling, was designed to produce porous poly(epsilon-caprolactone) scaffolds. The scaffold had a honeycomb-like pattern, fully interconnected channel network and controllable porosity and channel size [68]. In a recent study, Wu and colleagues presented a facile method using coaxial 3D printing strategy to prepare hollow strut-packed bioceramic scaffolds with designed macropores and multioriented hollow channels (Figure 6B) [69]. The hollow channels were not in the bulk of the porous scaffolds but in the struts. The unique hollow-struts structure of the bioceramic scaffold significantly improved cell attachment and proliferation and further promoted the formation of new bone tissue in the center of the scaffolds for large bone defects. Therefore, 3D bioprinting, as a new channel producing method, has become a very promising method to produce new channeled porous scaffolds.

From these studies, it can be seen that different approaches have their advantages and drawbacks (Table 2). According to different properties of the materials, an appropriate approach need to be considered to produce a channeled scaffold for a particular tissue regeneration application. 
Table 2. Fabrication approaches of channels.

\begin{tabular}{|lll|}
\hline Methods & Channel size & Advantages/drawbacks \\
\hline Removable template & $254,508 \mu \mathrm{m}$ & Ref. \\
\hline Templating rods & $1 \mathrm{~mm}$ & Controllable size/only straight, unbranched \\
\hline Micromold & $600 \mu \mathrm{m}$ & Cell embedded/complicated procedures \\
\hline Bottom-up & $900 \mu \mathrm{m}$ & Assemble cells into vascular-like shape/difficult to handle \\
\hline Sacrificial template & $1.16,0.60$ and $0.30 \mathrm{~mm}$ & Can make different channels with sizes and shapes/multiple steps \\
\hline Gel networks & Variable sizes & [59-62] \\
\hline 3D printing & & $\begin{array}{l}\text { Vascular-like branched networks/uncompleted removal of the } \\
\text { [6acrifice gels }\end{array}$ \\
\hline
\end{tabular}

\section{Conclusion \& future perspective}

Sufficient and rapid vascularization of synthetic porous scaffolds is still the key challenge in tissue engineering. Numerous approaches have been developed in the last decade to overcome this problem. Although new technologies and remarkable intermediate aims have been recently achieved, the generation of a functional vascularized tissue is still an unreached vision. The development of the channels in a porous scaffold represents a breakthrough and holds a great promise for vascularization as a new player in vascularization of a scaffold. Channel as a biophysical cue promotes the nutrition transport and rapid removal of wastes, which accelerates cell growth and vascularization. Although many efforts on the development of new reproducible and facile technologies are still needed, new 3D bioprinting technology or the combination of other approaches would bring new promising potentials to create porous scaffolds with vascular-like biomimetic channel structure for functional vascularization.

\section{Executive summary}

- Many strategies had been developed to address the key challenge.

- Channels in porous scaffolds provide a physical cue for vascularization.

- Channels promoted cell migration, proliferation and infiltration.

- Channels opened unblocked paths and provided spatial paths to promote nutrients transportation and blood supply, thus facilitating the vascularization of a porous scaffold.

- Numerous approaches have been developed to fabricate channels or microchannels in a porous scaffold.

- Mechanically removable template is the most extensively used approach to make micro-/macrochannels in a porous scaffold, but it is challenging to make branched channels.

- Sacrifice template is a facile method to make branched microchannels.

- 3D bioprinting technologies have been developed to print complex microchannels in scaffolds. 3D printing techniques not only produce branched, complex sacrifice templates for channels but also print channeled scaffolds directly.

- Channels can promote vascularization, which will bring a promising potential for tissue regeneration.

- With the combination of other players in vascularization, micro-/macrochannels become a new player in vascularization of tissue-engineered scaffolds.

Financial \& competing interests disclosure

The authors acknowledge the financial supported from the Osteo Science Foundation Philip J Boyne Junior Faculty Research Award. The authors have no other relevant affiliations or financial involvement with any organization or entity with a financial interest in or financial conflict with the subject matter or materials discussed in the manuscript apart from those disclosed.

No writing assistance was utilized in the production of this manuscript.

\section{Open Access}

This work is licensed under the Attribution-NonCommercial-NoDerivatives 4.0 Unported License. To view a copy of this license, visit http://creativecommons.org/licenses/by-nc-nd/4.0/ 


\section{References}

Papers of special note have been highlighted as: $\bullet$ of interest

1. De Long WG, Jr., Einhorn TA, Koval Ket al. Bone grafts and bone graft substitutes in orthopaedic trauma surgery. A critical analysis. J. Bone Joint Surg. Am. 89(3), 649-658 (2007).

2. Calori GM, Mazza E, Colombo M, Ripamonti C. The use of bone-graft substitutes in large bone defects: any specific needs? Injury 42(Suppl. 2) S56-S63 (2011).

3. Salgado AJ, Coutinho OP, Reis RL. Bone tissue engineering: state of the art and future trends. Macromol. Biosci. 4(8), 743-765 (2004).

4. Wang L, Fan H, Zhang ZY et al. Osteogenesis and angiogenesis of tissue-engineered bone constructed by prevascularized beta-tricalcium phosphate scaffold and mesenchymal stem cells. Biomaterials 31(36), 9452-9461 (2010).

5. Bi L, Cheng W, Fan H, Pei G. Reconstruction of goat tibial defects using an injectable tricalcium phosphate/chitosan in combination with autologous platelet-rich plasma. Biomaterials 31(12), 3201-3211 (2010).

6. Rezwan K, Chen QZ, Blaker JJ, Boccaccini AR. Biodegradable and bioactive porous polymer/inorganic composite scaffolds for bone tissue engineering. Biomaterials 27(18), 3413-3431 (2006).

7. Novosel EC, Kleinhans C, Kluger PJ. Vascularization is the key challenge in tissue engineering. Adv. Drug Deliv. Rev. 63(4-5), 300-311 (2011).

- Reviewed the key challenges in tissue engineering.

8. Laschke MW, Menger MD. Vascularization in tissue engineering: angiogenesis versus inosculation. Eur. Surg. Res. 48(2), 85-92 (2012).

9. Takebe T, Koike N, Sekine K et al. Generation of functional human vascular network. Transplant. Proc. 44(4), 1130-1133 (2012).

10. Zhao X, Liu L, Wang J et al. In vitro vascularization of a combined system based on a 3D printing technique. J. Tissue Eng. Regen. Med. 10(10), 833-842 (2014).

11. Nishiguchi A, Matsusaki M, Asano Y, Shimoda H, Akashi M. Effects of angiogenic factors and 3D-microenvironments on vascularization within sandwich cultures. Biomaterials 35(17), 4739-4748 (2014).

12. Mcguigan AP, Sefton MV. Vascularized organoid engineered by modular assembly enables blood perfusion. Proc. Natl Acad. Sci. USA 103(31), 11461-11466 (2006).

13. Chiu LL, Montgomery M, Liang Y, Liu H, Radisic M. Perfusable branching microvessel bed for vascularization of engineered tissues. Proc. Natl Acad. Sci. USA 109(50), E3414-E3423 (2012).

14. Nillesen ST, Geutjes PJ, Wismans R, Schalkwijk J, Daamen WF, Van Kuppevelt TH. Increased angiogenesis and blood vessel maturation in acellular collagen-heparin scaffolds containing both FGF2 and VEGF. Biomaterials 28(6), 1123-1131 (2007).

15. Wernike E, Montjovent MO, Liu Y et al. VEGF incorporated into calcium phosphate ceramics promotes vascularisation and bone formation in vivo. Eur. Cells Mater. 19, 30-40 (2010).

16. Unger RE, Ghanaati S, Orth C et al. The rapid anastomosis between prevascularized networks on silk fibroin scaffolds generated in vitro with cocultures of human microvascular endothelial and osteoblast cells and the host vasculature. Biomaterials 31(27), 6959-6967 (2010).

17. Deb S, Mandegaran R, Di Silvio L. A porous scaffold for bone tissue engineering/45S5 bioglass derived porous scaffolds for co-culturing osteoblasts and endothelial cells. J. Mater. Sci. Mater. Med. 21(3), 893-905 (2010).

18. Liu Y, Teoh SH, Chong MS et al. Contrasting effects of vasculogenic induction upon biaxial bioreactor stimulation of mesenchymal stem cells and endothelial progenitor cells cocultures in three-dimensional scaffolds under in vitro and in vivo paradigms for vascularized bone tissue engineering. Tissue Eng. Part A 19(7-8), 893-904 (2013).

19. Baranski JD, Chaturvedi RR, Stevens KR et al. Geometric control of vascular networks to enhance engineered tissue integration and function. Proc. Natl Acad. Sci. USA 110(19), 7586-7591 (2013).

20. Shin M, Matsuda K, Ishii $\mathrm{O}$ et al. Endothelialized networks with a vascular geometry in microfabricated poly(dimethyl siloxane). Biomed. Microdevices 6(4), 269-278 (2004).

21. Chang R, Nam J, Sun W. Effects of dispensing pressure and nozzle diameter on cell survival from solid freeform fabrication-based direct cell writing. Tissue Eng. Part A 14(1), 41-48 (2008).

22. Carmeliet P, Jain RK. Angiogenesis in cancer and other diseases. Nature 407(6801), 249-257 (2000).

23. Kannan RY, Salacinski HJ, Sales K, Butler P, Seifalian AM. The roles of tissue engineering and vascularisation in the development of micro-vascular networks: a review. Biomaterials 26(14), 1857-1875 (2005)

24. Jain RK, Au P, Tam J, Duda DG, Fukumura D. Engineering vascularized tissue. Nat. Biotechnol. 23(7), 821-823 (2005).

25. Levenberg S, Rouwkema J, Macdonald M et al. Engineering vascularized skeletal muscle tissue. Nat. Biotechnol. 23(7), 879-884 (2005).

26. Langer R, Vacanti JP. Tissue engineering. Science 260(5110), 920-926 (1993).

27. Radisic M, Park H, Shing $\mathrm{H}$ et al. Functional assembly of engineered myocardium by electrical stimulation of cardiac myocytes cultured on scaffolds. Proc. Natl Acad. Sci. USA 101(52), 18129-18134 (2004). 
28. Zimmermann WH, Schneiderbanger K, Schubert $\mathrm{P}$ et al. Tissue engineering of a differentiated cardiac muscle construct. Circ. Res. 90(2), 223-230 (2002).

29. Murphy CL, Sambanis A. Effect of oxygen tension on chondrocyte extracellular matrix accumulation. Connect. Tissue Res. 42(2), 87-96 (2001).

30. Murphy CL, Polak JM. Control of human articular chondrocyte differentiation by reduced oxygen tension. J. Cell. Physiol. 199(3), 451-459 (2004).

31. Mofrad AZ, Mashayekhan S, Bastani D. Simulation of the effects of oxygen carriers and scaffold geometry on oxygen distribution and cell growth in a channeled scaffold for engineering myocardium. Math. Biosci. 294, 160-171 (2017).

32. Radisic M, Deen W, Langer R, Vunjak-Novakovic G. Mathematical model of oxygen distribution in engineered cardiac tissue with parallel channel array perfused with culture medium containing oxygen carriers. Am. J. Physiol. Heart Circ. Physiol. 288(3), H1278-H1289 (2005).

33. Song YS, Lin RL, Montesano G et al. Engineered 3D tissue models for cell-laden microfluidic channels. Anal. Bioanal. Chem. 395(1), 185-193 (2009).

- Used tissue models to elucidate the importance of the delivery of nutrients and oxygen within 3D tissue constructs to maintain cell viability.

34. Truslow JG, Price GM, Tien J. Computational design of drainage systems for vascularized scaffolds. Biomaterials 30(26), 4435-4443 (2009).

35. Takei T, Kitazono Z, Ozuno Y, Yoshinaga T, Nishimata H, Yoshida M. Vascular-like network prepared using hollow hydrogel microfibers. J. Biosci. Bioeng. 121(3), 336-340 (2016).

36. Stosich MS, Bastian B, Marion NW, Clark PA, Reilly G, Mao JJ. Vascularized adipose tissue grafts from human mesenchymal stem cells with bioactive cues and microchannel conduits. Tissue Eng. 13(12), 2881-2890 (2007).

37. Tansey KE, Seifert JL, Botterman B, Delgado MR, Romero MI. Peripheral nerve repair through multi-luminal biosynthetic implants. Ann. Biomed. Eng. 39(6), 1815-1828 (2011).

38. Bertassoni LE, Cecconi M, Manoharan V et al. Hydrogel bioprinted microchannel networks for vascularization of tissue engineering constructs. Lab on a Chip 14(13), 2202-2211 (2014).

39. Kang Y, Mochizuki N, Khademhosseini A, Fukuda J, Yang Y. Engineering a vascularized collagen-beta-tricalcium phosphate graft using an electrochemical approach. Acta Biomater. 11, 449-458 (2015).

- Reported an electrochemical approach to produce microchannel hydrogel with cells in a macrochanneled porous ceramic scaffold.

40. Kageyama T, Kakegawa T, Osaki T et al. Rapid engineering of endothelial cell-lined vascular-like structures in in situ crosslinkable hydrogels. Biofabrication 6(2), 025006 (2014).

\section{- Reported a facile technique to align endothelial cells with microchannels in a hydrogel.}

41. Arrigoni C, Bongio M, Talo G et al. Rational design of prevascularized large 3D tissue constructs using computational simulations and biofabrication of geometrically controlled microvessels. Adv. Healthcare Mater. 5(13), 1617-1626 (2016).

42. Li S, Liu Y, Li Y, Liu C, Sun Y, Hu Q. A novel method for fabricating engineered structures with branched micro-channel using hollow hydrogel fibers. Biomicrofuidics 10(6), 064104 (2016).

43. Tseng TC, Hsieh FY, Theato P, Wei Y, Hsu SH. Glucose-sensitive self-healing hydrogel as sacrificial materials to fabricate vascularized constructs. Biomaterials 133, 20-28 (2017).

44. Justin AW, Brooks RA, Markaki AE. Multi-casting approach for vascular networks in cellularized hydrogels. J. R. Soc. Interface 13(125), pii: 20160768 (2016).

45. Lee VK, Lanzi AM, Haygan N, Yoo SS, Vincent PA, Dai G. Generation of multi-scale vascular network system within 3D hydrogel using 3D bio-printing technology. Cell. Mol. Bioeng. 7(3), 460-472 (2014).

46. Huang H, Oizumi S, Kojima N, Niino T, Sakai Y. Avidin-biotin binding-based cell seeding and perfusion culture of liver-derived cells in a porous scaffold with a three-dimensional interconnected flow-channel network. Biomaterials 28(26), 3815-3823 (2007).

47. Yu T, Dong C, Shen $\mathrm{Z}$ et al. Vascularization of plastic calcium phosphate cement in vivo induced by in-situ-generated hollow channels. Mater. Sci. Eng. C Mater. Biol. Appl. 68, 153-162 (2016).

48. Varoni EM, Altomare L, Cochis A et al. Hierarchic micro-patterned porous scaffolds via electrochemical replica-deposition enhance neo-vascularization. Biomed. Mater. 11(2), 025018 (2016).

49. Zhang W, Wray LS, Rnjak-Kovacina J et al. Vascularization of hollow channel-modified porous silk scaffolds with endothelial cells for tissue regeneration. Biomaterials 56, 68-77 (2015).

50. Maidhof R, Marsano A, Lee EJ, Vunjak-Novakovic G. Perfusion seeding of channeled elastomeric scaffolds with myocytes and endothelial cells for cardiac tissue engineering. Biotechnol. Prog. 26(2), 565-572 (2010).

51. Rose FR, Cyster LA, Grant DM, Scotchford CA, Howdle SM, Shakesheff KM. In vitro assessment of cell penetration into porous hydroxyapatite scaffolds with a central aligned channel. Biomaterials 25(24), 5507-5514 (2004). 
52. Yu T, Liu Q, Jiang T, Wang X, Yang Y, Kang Y. Channeled $\beta$-TCP scaffolds promoted vascularization and bone augmentation in mandible of Beagle dogs. Adv. Funct. Mater. 26(37), 6719-6727 (2016).

53. Rnjak-Kovacina J, Wray LS, Golinski JM, Kaplan DL. Arrayed hollow channels in silk-based scaffolds provide functional outcomes for engineering critically-sized tissue constructs. Adv. Funct. Mater. 24(15), 2188-2196 (2014).

- Reported the critical function of hollow channels in promoting functional vascularization.

54. Wray LS, Rnjak-Kovacina J, Mandal BB, Schmidt DF, Gil ES, Kaplan DL. A silk-based scaffold platform with tunable architecture for engineering critically-sized tissue constructs. Biomaterials 33(36), 9214-9224 (2012).

55. Osaki T, Kakegawa T, Mochizuki N, Fukuda J. Fabrication of perfusable vasculatures by using micromolding and electrochemical cell transfer. Conf. Proc. IEEE Eng. Med. Biol. Soc. 2013, 6655-6658 (2013).

56. Sadr N, Zhu M, Osaki T et al. SAM-based cell transfer to photopatterned hydrogels for microengineering vascular-like structures. Biomaterials 32(30), 7479-7490 (2011).

57. Osaki T, Kakegawa T, Kageyama T, Enomoto J, Nittami T, Fukuda J. Acceleration of vascular sprouting from fabricated perfusable vascular-like structures. PLoS ONE 10(4), e0123735 (2015).

58. Du Y, Ghodousi M, Qi H, Haas N, Xiao W, Khademhosseini A. Sequential assembly of cell-laden hydrogel constructs to engineer vascular-like microchannels. Biotechnol. Bioeng. 108(7), 1693-1703 (2011).

59. Hirama H, Odera T, Torii T, Moriguchi H. A lithography-free procedure for fabricating three-dimensional microchannels using hydrogel molds. Biomed. Microdevices 14(4), 689-697 (2012).

60. Huang GY, Zhou LH, Zhang QC et al. Microfluidic hydrogels for tissue engineering. Biofabrication 3(1), 012001 (2011).

61. Guo Z, Higgins CA, Gillette BM et al. Building a microphysiological skin model from induced pluripotent stem cells. Stem Cell. Res. Ther. 4(Suppl. 1), S2 (2013).

62. Chan HN, Shu Y, Tian Q, Chen Y, Chen Y, Wu H. Replicating 3D printed structures into hydrogels. Mater. Horiz. 3(4), 309-313 (2016).

63. Golden AP, Tien J. Fabrication of microfluidic hydrogels using molded gelatin as a sacrificial element. Lab on a Chip 7(6), 720-725 (2007).

64. Hu C, Sun H, Liu Z et al. Freestanding 3-D microvascular networks made of alginate hydrogel as a universal tool to create microchannels inside hydrogels. Biomicrofluidics 10(4), 044112 (2016).

65. Li S, Liu YY, Liu LJ, Hu QX. A versatile method for fabricating tissue engineering scaffolds with a three-dimensional channel for prevasculature networks. ACS Appl. Mater. Interfaces 8(38), 25096-25103 (2016).

66. Gao Q, He Y, Fu JZ, Liu A, Ma L. Coaxial nozzle-assisted 3D bioprinting with built-in microchannels for nutrients delivery. Biomaterials 61, 203-215 (2015).

67. Becker ST, Bolte H, Krapf O et al. Endocultivation: 3D printed customized porous scaffolds for heterotopic bone induction. Oral Oncol. 45(11), e181-e188 (2009).

68. Zein I, Hutmacher DW, Tan KC, Teoh SH. Fused deposition modeling of novel scaffold architectures for tissue engineering applications. Biomaterials 23(4), 1169-1185 (2002).

69. Luo $\mathrm{Y}$, Zhai $\mathrm{D}$, Huan $\mathrm{Z}$ et al. Three-dimensional printing of hollow-struts-packed bioceramic scaffolds for bone regeneration. ACS Appl. Mater. Interfaces 7(43), 24377-24383 (2015).

- Published a new 3D printing technique to produce channeled ceramic scaffolds for bone tissue regeneration. 
